\title{
画HAD
}

ISSN-L: 2530-5115

DOI: http://doi.org/10.22585/hospdomic.v1i2.11

\section{Evaluación de la calidad de las páginas Web sobre el Hospital a Domicilio: el Indicador de Credibilidad como factor pronóstico}

Evaluating the quality of Websites related to Hospital-Based Home Care: The Credibility Indicator as a prognostic factor

María Sanz-Lorentel', Rocio Guardiola-Wanden-Berghe ${ }^{2,3}$

1. Centro de Salud Pública de la Consellería de Sanidad Universal y Salud Pública. Manises, España.

2. Instituto de Neuropsiquiatría y Adicciones (INAD), Consorci Parc de Salut Mar. Barcelona, España.

3. Centro de Investigación Biomédica. Barcelona, España.

Correspondencia/Correspondence

msanzlor@gmail.com

Recibido/Received

26.02.2017

Aceptado/Accepted

19.03.2017
Conflicto de Intereses/Competing interest No existe ningún conflicto de interés en el presente estudio.

CÓMO CITAR ESTE TRABAJO | HOW TO CITE THIS PAPER

Sanz-Lorente M, Guardiola-Wanden-Berghe R. Evaluación de la calidad de las páginas Web sobre el Hospital a Domicilio: el Indicador de Credibilidad como factor pronóstico. Hosp Domic. 2017; 1 (2):7382. 


\section{RESUMEN}

Objetivo: Evaluar la calidad documental de las páginas Web relacionadas con el Hospital a Domicilio.

Método: Estudio transversal de los Websites sobre Hospital a Domicilio, accediendo a la población a estudio a través de la búsqueda en Google, teniendo en cuenta la "falacia muestral" de este buscador. La calidad se estudió utilizando las 8 variables pertenecientes al Indicador de Credibilidad (IC).

Resultado: Se evaluaron un total de 215 páginas activas, que pertenecían principalmente a medios de comunicación. No se encontró ninguna Web que cumpliera con los 8 criterios del IC; obteniéndose Media de 2,12 \pm 0,07; Mínimo de 0 y Máximo de 5; Mediana igual a 3. De las Webs estudiadas, 74 (34,42\%) presentaban simultáneamente autoría y filiación, existiendo asociación con el cumplimiento del IC con las páginas que disponían de estas 2 variables ( $p$ $<0,001)$.

Conclusiones: Existió una baja calidad de las páginas Web relacionadas con el Hospital a Domicilio. En este estudio se confirmó que la existencia de autoría y filiación fueron un factor pronóstico de la calidad de las Webs analizadas. El Indicador de Credibilidad fue una ayuda a la hora de conocer la calidad de un sitio Web.

Palabras clave: Servicios de Atención a Domicilio Provisto por Hospital; Comunicación en Salud; Internet; Control de Calidad; Diseminación de Información.

\section{ABSTRACT}

Objective: To evaluate the documental quality of websites related to Home Care Services.

Method: This is a descriptive cross-sectional study of websites based on Home Care Services, using searches on Google to access the study population. The "fallacy sample" of this search engine was take into account. The quality was studied thought the 8 variables of the Credibility Indicator $(\mathrm{Cl})$.

Results: A total of 215 active websites, mainly belonging to the media, were evaluated. None of the websites met all 8 items in the $\mathrm{Cl}$. Mean of 2,12 $\pm 0,07$; Minimum of 0 and Maximum of 5 ; Median equal to 3 . Within the studied websites, $74(34,42 \%)$ presented both authorship and affiliation. There was an association between the $\mathrm{Cl}$ accomplishment and websites that had these 2 variables $(p<0.001)$.

Conclusions: The quality of websites covering issues of Hospital-Based Home Care services is still poor. It is confirmed that identifying authorship and affiliation is an important factor in predicting the quality of the information. The Credibility Indicator is a useful aid when determining the quality of a website.

Keywords: Home Care Services, Hospital-Based; Health Communication; Internet; Quality Control; Information Dissemination. 


\section{INTRODUCCIÓN}

Internet es sin duda el mayor repositorio de información, al que una población cada vez más familiarizada con las nuevas tecnologías de la comunicación y la información, puede acceder fácilmente. No es desatinado calificar a la Web como el principal medio donde conseguir información sobre salud, permitiendo aproximarse sin restricciones a casi toda la información susceptible de ser transmitida (1). Esta circunstancia, aconsejaría contar con criterios, de fácil uso, que permitan identificar la información de calidad.

En este sentido, se han propuesto diferentes enfoques desde los que abordar la evaluación de la calidad de las páginas Web. Algunas propuestas han desarrollado modelos basados en metodologías ya usadas en otros contextos (2). Otros trabajos han refundido las variables propuestas por entidades internacionales de evaluación y/o acreditación de páginas Web (3). Y, algunas organizaciones han proporcionado herramientas específicas para la búsqueda, calificación y clasificación de la información sanitaria (4). El fin de estas herramientas es ayudar a las personas a filtrar los mensajes válidos y fiables de los inexactos o engañosos.

Para lograr este fin, la Comisión Europea (5) propuso una serie de recomendaciones para la consecución de un amplio consenso para conseguir una calidad apropiada en relación a los sitios Web sobre salud: Aplicar los criterios de calidad relacionados con la salud de una manera apropiada al sitio Web y a los usuarios; desplegar campañas de información para educar a los desarrolladores de sitios y a los ciudadanos sobre los niveles mínimos de calidad; aprovechar la gran información sobre salud que ofrece la Unión Europea y otras instituciones en beneficio de los ciudadanos (traducción y adaptación cultural); promover el intercambio de información y experiencias a nivel europeo sobre la manera de aplicar los criterios de calidad.

Es evidente que disponer de indicadores para aplicar en el proceso de evaluación es, sin lugar a dudas, necesario. Aun así, muchas veces los mismos son de difícil comprensión por aquellas personas no expertas en la materia y que, al fin y al cabo, son los usuarios finales de la ingente información disponible en la Red $(6,7)$.

Con el fin de facilitar la tarea de comprobación de la calidad Web, existen estudios que han intentado simplificar y unificar las variables a testar. Por ejemplo, Lopes (8) concentró en 7 categorías los indicadores de calidad con el fin de determinar la credibilidad de la información sobre salud en la Web. Posteriormente, esta misma autora propuso que para observar patrones de calidad sería necesario utilizar criterios de credibilidad del sitio Web y también los de contenido (3).

A partir de estas propuestas, Guardiola-Wanden-Berghe et al (9), refundieron en 22 ítems las variables sobre calidad Web aportadas por las principales instituciones, demostrando a su vez una correlación positiva entre el cumplimiento de las variables de calidad y el Indicador de Credibilidad (8 ítems). Esta correspondencia daba al usuario general la posibilidad de valorar la calidad de una determinada Web utilizando tan solo 8 variables de fácil comprensión.

Por todo lo anteriormente expuesto, este trabajo tiene como objetivo evaluar, mediante el Indicador de Credibilidad, la calidad documental de las páginas Web relacionadas con el Hospital a Domicilio.

\section{MATERIAL Y MÉTODO}

\section{Diseño:}

Estudio descriptivo trasversal, en el que la población a estudio son las páginas Web, resultantes de su acceso, desde la referencia obtenida de la búsqueda en Google España [http://www.google. es/]. 
Fuente de obtención de datos:

Se obtuvieron de la consulta directa y acceso, vía Internet, a las Webs motivo de estudio. La búsqueda se realizó mediante la ecuación, insertada de la siguiente forma:

\section{| "hospitalización a domicilio" | "hospitalización en casa" | "hospitalización domiciliaria" | "hospital a domicilio" |}

Para evitar modificaciones en los resultados y mantener el Posicionamiento resultante, se almacenaron las referencias obtenidas en la búsqueda en formato pdf, con hipervínculo en cada referencia, para volver a acceder a la página Web en cualquier momento.

Se consideró la «Falacia Muestral de Google». Este buscador nunca ofrece un resultado mayor al millar de Websites. Pero, "estima" el número de páginas Web que existen en los servidores rastreados por su robot de exploración "Googlebot or Spider», en relación con el tiempo empleado en reconocer el primer millar.

\section{Criterios de exclusión:}

Referencias a documentos Portable Document Format (pdf), a materiales multimedia (video) o que la dirección Uniform Resource Locator (URL) fuera inexistente o el enlace estuviera roto.

Tratamiento de la información:

Para el almacenamiento de los datos y los cálculos estadísticos, se empleó el programa SPSS $®$ versión 22 para Windows. Se cumplimentaron dobles tablas, para posteriormente comparar la igualdad entre las mismas y así evitar errores de transcripción.

Variables a estudio:

1. Ítems pertenecientes al Indicador de Credibilidad (IC).

- Autoría - Persona o personas responsable/s de los contenidos.

- Filiación - Organismo, relacionado con el autor, que ampara el sitio Web (su origen y/o responsabilidad).

- Equipo editorial - Responsables de la línea editorial y/o contenido de la Web.

- Fecha de creación - Fecha en la que se realizó por primera vez la Web.

- Fecha de actualización - Fecha de la última modificación.

- Aval - Existencia de garantía de instituciones profesionales o académicas relevantes (consecuentemente, a las sociedades científicas se les asignó siempre este ítem).

- Acreditación - Adopción de códigos de calidad y conducta ética de organismos oficiales de certificación y normalización.

- Financiación - Reconocimiento explícito de cualquier patrocinio o mecenazgo relacionado con la Web y/o de las personas responsables.

2. Otras variables.

- Página activa - Validez o funcionamiento del enlace desde la referencia a la página Web.

- Institución - Tipo de organismo público o privado propietario de la página Web.

\section{Análisis de los datos:}

Las variables cuantitativas se describieron con su Media y Desviación Estándar y las cualitativas con su valor absoluto y porcentaje. Se utilizó la Media y la Mediana como medidas de tendencia 
central. Para comprobar la significación de la diferencia de medias para muestras independientes se utilizó la prueba t de Student. La existencia de asociación entre variables cualitativas se analizó mediante la prueba de Chi-cuadrado de Pearson. El nivel de significación utilizado en todos los contrastes de hipótesis fue $a \leq 0,05$. Algunas variables se representaron utilizando tablas o figuras.

\section{RESULTADOS}

A partir de la búsqueda realizada se pudieron obtener un total de 251 referencias, de las cuales $215(85,66 \%)$ correspondieron a páginas activas, eliminándose 29 (11,55\%) por ser documentos pdf y 1 por ser un video; $6(2,39 \%)$ referencias presentaron su enlace roto.

Las Webs analizadas pertenecían principalmente a medios de comunicación y a entidades privadas, en 4 (1,86\%) páginas no se pudo determinar la entidad responsable; ver tabla 1.

\begin{tabular}{|c|c|c|}
\hline Tipo de institución & Frecuencia & Porcentaje \\
\hline Medio de comunicación & 70 & 32,56 \\
\hline Entidad privada & 69 & 32,09 \\
\hline Institución pública & 29 & 13,49 \\
\hline Editorial científica & 26 & 12,09 \\
\hline Sociedad científica & 10 & 4,65 \\
\hline Web personal & 7 & 3,26 \\
\hline No consta & 4 & 1,86 \\
\hline Total & 215 & 100,00 \\
\hline
\end{tabular}

El análisis descriptivo del cumplimiento de las variables del Indicador de Credibilidad (IC) presentó los siguientes resultados: Media de 2,12 \pm 0,07; Mínimo de 0 y Máximo de 5; Mediana igual a 3. No se encontró ninguna página Web que cumpliera con los 8 criterios de calidad. Los cumplimientos de cada uno de estos criterios en el total de Webs estudiadas pueden consultarse en la tabla 2. Los resultados sobre el cumplimiento del IC segregándolos por tipo de institución mostraron que el mayor cumplimiento se daba en las páginas de las editoriales científicas; ver tabla 3. La relación de estos datos con el valor de la Mediana del total de Webs analizadas se puede observar en la figura 1. 


\begin{tabular}{|c|c|c|}
\hline Ítem del I.C. & Frecuencia & Porcentaje \\
\hline Autoría & 84 & 39,07 \\
\hline Filiación & 183 & 85,12 \\
\hline Equipo editorial o responsable & 22 & 10,23 \\
\hline Fecha de creación & 144 & 66,98 \\
\hline Fecha de actualización & 5 & 2,33 \\
\hline Aval & 12 & 5,58 \\
\hline Acreditación & 4 & 1,86 \\
\hline Financiación & 2 & 0,93 \\
\hline
\end{tabular}

\begin{tabular}{|l|c|c|c|c|}
\hline \multicolumn{6}{|c|}{ Tabla 3. Cumplimiento del Indicador de Credibilidad según tipo de institución. } \\
\hline Tipo de institución & Media & Mediana & Máximo & Mínimo \\
\hline Medio de comunicación & $2,49 \pm 0,09$ & 2 & 4 & 1 \\
\hline Entidad privada & $1,49 \pm 0,11$ & 1 & 3 & 0 \\
\hline Institución pública & $1,96 \pm 0,20$ & 2 & 4 & 1 \\
\hline Editorial científica & $2,92 \pm 0,10$ & 3 & 4 & 2 \\
\hline Sociedad científica & $2,90 \pm 0,31$ & 3 & 5 & 1 \\
\hline Web personal & $2,00 \pm 0,31$ & 2 & 3 & \\
\hline
\end{tabular}


Figura 1. Cumplimiento del Indicador de Credibilidad según tipo de institución y su relación con el valor de la Mediana del total de Webs sobre Hospital a Domicilio analizadas.

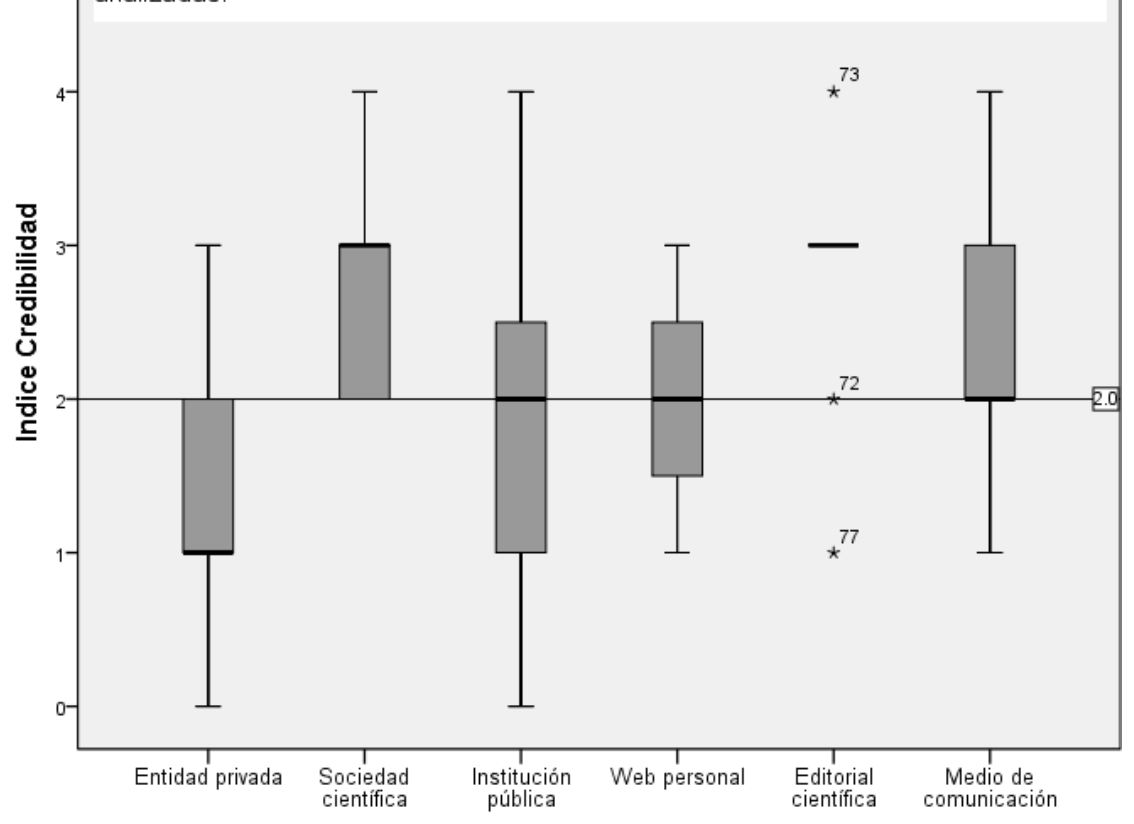

Institucion

Del total de Webs estudiadas, 74 (34,42\%) presentaban simultáneamente autoría y filiación, existiendo diferencias significativas en el cumplimiento del IC entre las páginas que disponían de estas variables y las que no (prueba t para muestras independientes igual a $-17,72$, con 206,79 g.l. y $p<0,001)$; ver tabla 4.

Tabla 4. Cumplimiento del Indicador de Credibilidad según exista, o no, autoría y filiación.

\begin{tabular}{|l|c|c|c|c|}
\hline Autoría y filiación & Media & Mediana & Máximo & Mínimo \\
\hline $\mathrm{Si}$ & $3,08 \pm 0,04$ & 3 & 4 & 2 \\
\hline No & $1,62 \pm 0,07$ & 2 & 5 & 0 \\
\hline
\end{tabular}


La existencia de asociación entre la autoría y filiación, conjuntamente, con el resto de las variables que componen el IC, analizada mediante la prueba de la chi cuadrado puede consultarse en la tabla 5, donde se observa la asociación de estas dos variables conjuntas con la existencia de fecha de creación $(p<0,01)$.

\begin{tabular}{|c|c|c|}
\hline & Criterio del IC & Significación \\
\hline \multirow[t]{6}{*}{ Autoría y Filiación } & Equipo editorial o responsable & 0,81 \\
\hline & Fecha de creación & $<0,01$ \\
\hline & Fecha de actualización & 0,79 \\
\hline & Aval & 0,05 \\
\hline & Acreditación & 0,14 \\
\hline & Financiación & 0,64 \\
\hline
\end{tabular}

\section{DISCUSIÓN}

Los resultados obtenidos indicaron un pobre cumplimiento de los criterios relacionados con el Indicador de Credibilidad, siendo destacable que los valores de la Mediana en ningún caso alcanzara la mitad de los ítems del Indicador (8 ítems).

Los valores observados generaron la pregunta de cómo asegurar y juzgar la calidad de sus mensajes, creando incertidumbre sobre la información que se pudiera obtener de estas páginas Web.

El número de enlaces no activos, accesibilidad a las Webs, fue inferior al descrito en estudios anteriores (9), lo que permite aventurar una mayor supervivencia de los sites sobre el Hospital a Domicilio.

Internet ha cambiado muchos aspectos de la relación del ciudadano con la información y la gestión de su salud. Es reseñable que los medios de comunicación fueran el tipo de institución más representativo, ya que estos tienen impacto en la salud colectiva e individual y son fundamentales para conformar opinión. En el sector salud los medios de comunicación juegan un rol de gran importancia, ya que el contenido mediático crea y consolida conductas, creencias y valores (10). También, hay que tener en cuenta la presencia de editoriales científicas. Esta información es una buena fuente para el periodista, pues le proporciona veracidad y novedad (11). Si bien las sociedades científicas presentan los mejores resultados, el bajo cumplimiento de los criterios de calidad, representados por el Indicador de Credibilidad, obliga a una importante y urgente revisión y mejora de estas "sedes virtuales" al ser referentes sociales de la población que consulta sobre el Hospital 
a Domicilio. Esta circunstancia ya fue observada en otras sociedades científicas del área de las ciencias de la salud (12).

El incumplimiento de los criterios sobre la fecha de actualización, aval, acreditación y financiación, también ha sido observado en estudios previos $(9,13)$. La falta de atención por los responsables de las Webs coincide con el bajo uso y consulta, de estos ítems, por la población general. Pero, priva a quien accede a ellas de una importante información necesaria para poder conocer su credibilidad. Se sabe que los usuarios no suelen juzgar la calidad de la información sobre salud obtenida en la Red (14) o utilizan criterios poco válidos para juzgar la veracidad de la información o su actualidad (15).

Le relación de la autoría y filiación con el mayor cumplimiento del Indicador de Credibilidad puede ser un factor importante a la hora de predecir la calidad de la información, aunque no siempre se cumple esta premisa (16). Al menos, debemos tener presente que la presencia de autor ligado a una institución de referencia es un primer criterio de calidad a tener en cuenta. La Web está repleta de opiniones individuales y en muchos casos estas se disfrazan detrás de personajes inexistentes, falsos o que se ocultan en el anonimato de Internet.

En definitiva, está claro que la calidad se define a partir de las expectativas de los usuarios, lo que implica un componente subjetivo muy alto (17). De todos modos, contar con una sencilla ayuda para valorar un sitio Web de forma rápida y sencilla siempre será una ayuda. Como solicitaba Ferguson (18), disponer de un sencillo protocolo que permita aprovechar los recursos de Internet con unas mínimas garantías de veracidad. La regulación no parece ser la estrategia correcta para mejorar la calidad de la información de salud en Internet. Otros enfoques, como educar a los responsables de este contenido, parecen una mejor apuesta a largo plazo (19).

En consecuencia, se podría concluir que existió una baja calidad de las páginas Web relacionadas con el Hospital a Domicilio. En este estudio se demostró que la existencia de autoría y filiación fueron un factor pronóstico de la calidad de las Webs analizadas. El Indicador de Credibilidad fue una ayuda a la hora de conocer la calidad de un sitio Web.

\section{BIBLIOGRAFÍA}

1. Sanz-Valero J, Castiel LD, Wanden-Berghe C, Juan Quilis V. Internet y la búsqueda de información en salud pública: desde la relevancia hacia la «revelancia». Gac Sanit. 2006;20(2):159-60. DOI: 10.1157/13087329; PMID: 16753094

2. Herrera-Viedma E, Pasi G, Lopez-Herrera AG, Porcel C. Evaluating the information quality of Web sites: A methodology based on fuzzy computing with words. J Am Soc Inf Sci Technol. 2006;57(4):538-49. DOI: 10.1002/asi.20308

3. Lopes IL. Criterios de qualidade para avaliaçao da informaçao na World Wide Web. Brasilia, Brasil: Editora do Departamento de Ciencia da Informaçao da Universidade de Brasilia; 2007.

4. Gil Pérez JD. Internet como generador de opinión en la juventud española: valoración de la calidad y credibilidad de las páginas Web más consultadas por lo jóvenes españoles [tesis doctoral]. Alicante, España: Universidad de Alicante; 2011.

5. Commission of the European Communities. eEurope 2002: Quality criteria for health related Websites. J Med Internet Res. 2002;4(3):e15. DOI: 10.2196/jmir.4.3.e15; PMID: 12554546

6. Eysenbach G, Powell J, Kuss O, Sa E-R. Empirical studies assessing the quality of health information for consumers on the world wide web: a systematic review. JAMA. 2002;287(20):2691700. DOI: 10.1001/jama.287.20.2691; PMID: 12020305 
7. Guardiola-Wanden-Berghe R, Sanz-Valero J, Wanden-Berghe C. Eating disorders blogs: testing the quality of information on the internet. Eat Disord. 2010;18(2):148-52. DOI: 10.1080/10640260903585565; PMID: 20390618

8. Lopes IL. Novos paradigmas para avaliação da qualidade da informação em saúde recuperada na Web. Ciênc Informação. 2004;33(1):81-90. DOI: 10.1590/S0100-19652004000100010

9. Guardiola-Wanden-Berghe R, Gil-Pérez JD, Sanz-Valero J, Wanden-Berghe C. Evaluating the quality of websites relating to diet and eating disorders. Health Inf Libr J. 2011;28(4):294-301. DOI: 10.1111/j.1471-1842.2011.00961.x; PMID: 22051128

10. Feo Acevedo C, Feo Istúriz O. Impacto de los medios de comunicación en la salud pública. Saúde Em Debate. 2013;37(96):84-95.

11. Revuelta G, de Semir V, editores. Medicina y Salud en la prensa diaria: Informe Quiral 10 años. Barcelona, España: Observatorio de la Comunicación Científica, Universitat Pompeu Fabra; 2008.

12. Sanz Valero J, Wanden-Berghe C, Guardiola-Wanden-Berghe R, Culebras Fernández JM. Credibilidad de las páginas Web de las sociedades científicas sobre nutrición y alimentación. Nutr Hosp. 2010;25(Supl 2):62-3.

13. Guardiola-Wanden-Berghe R, Sanz Valero J, Wanden-Berghe C. Aportaciones a la evaluación de la calidad de páginas web sobre trastornos de la conducta alimentaria como pronóstico de la aptitud de su información. Nutr Hosp. 2011;26(Supl 1):77.

14. Jiménez Pernett J, García Gutiérrez JF, Bermúdez Tamayo C, Silva Castro MM, Tuneu i Valls L. Evaluación de sitios web con información sobre medicamentos. Aten Primaria. 2009;41(7):3606. DOI: 10.1016/j.aprim.2008.10.009; PMID: 19464077

15. Rice RE. Influences, usage, and outcomes of Internet health information searching: multivariate results from the Pew surveys. Int J Med Inf. 2006;75(1):8-28. DOI: 10.1016/j.ijmedinf.2005.07.032; PMID: 16125453

16. Oller-Arlandis VE, Sanz Valero J. Autoría y filiación como indicador pronóstico de la calidad en la Web 2.0: los Blogs sobre medio ambiente. JONNPR. 2017;2. DOI: http://dx.doi.org/10.19230/ jonnpr.1311

17. Ayuso García MD, Martínez Navarro V. Evaluación de calidad de fuentes y recursos digitales: Guía de buenas prácticas. An Doc. 2006;(9):17-42.

18. Ferguson T. From patients to end users. BMJ. 2002;324(7337):555-6. DOI: 10.1136/ bmj.324.7337.555; PMID: 11884301

19. Purcell GP. The quality of health information on the internet. BMJ. 2002;324(7337):557-8. DOI: 10.1136/bmj.324.7337.557; PMID: 11884303 